\title{
Using Facial Tissue Expanders in Repositioning of Linear and Irregular Cheek Scars for the Quest of Superior Aesthetic Results
}

\author{
MOHAMED A. WAHSH, M.D. and MAHFOUZ SHEHATA, M.D., M.R.C.S. \\ The Department of General Surgery, Plastic Surgery Unit, Faculty of Medicine, Zagazig University, Egypt
}

\begin{abstract}
Background: The motive of this study was that the results of some cheek scars revisions may appear to be disappointing owing to their sites or direction even with much efforts. By using tissue expanders, the scars repositioning and reorientation in relation to the cheek unit borders and Resting Skin Tension Lines (RSTLs) may provide the basis of superior results in scar revision and management. So, we aim to demonstrate the effect of cheek skin expansion by tissue expanders on scar repositioning and reorientation to achieve a superior aesthetic outcome.
\end{abstract}

Methods: This prospective study was conducted on 13 patients with obvious linear (8 cases) or irregular (5 cases) cheek scars who were willing to move these scars to less conspicuous sites. We used rectangular tissue expanders in all cases with ( 40 to $200 \mathrm{ml}$.) capacity. They were selected suitable to be inserted under the donor tissue and generate the proposed expansion required for scar repositioning.

Results: The concept of complete moving the scar outside the cheek unit (optimal revision) has been applicable to 5 in 13 patients $(38.5 \%)$. However, suboptimal revision has been applicable in the form of scar reorientation typically in RSTLs succeeded in 5 of 13 patients $(38.5 \%)$ or repositioning the scar outside the malar region in one case $(7.7 \%)$. Partial revision in the form of incomplete scar resection occurred in 1 case $(7.7 \%)$. Only in one case $(7.7 \%)$, the expansion was aborted due to early wound dehiscence (failure). In 10 out of 13 tissue expansion cases $(77 \%)$ were achieved without complications. At the same time, one minor complication has occurred in one patient i.e. hematoma (7.7\%). Major complications have occurred in 2 cases i.e. expanders were exposed $(15.4 \%)$.

Conclusion: Facial tissue expander usage in repositioning and revision of linear and irregular cheek scar is a reasonable approach in obtaining superior aesthetic results.

Key Words: Cheek - Scar - Repositioning - Expander Aesthetic-Subunit.

\section{INTRODUCTION}

The face has its great importance in giving the person his self-identity, beauty and individual characteristics. However, being an exposed and easily susceptible to trauma and wounds, it is the part which faces a great risk of injuries and as a result it resembles a common site of scars [1,2].
The patients suffering from scars are looking forward to restoring the pre-injury look and becoming again without scar which is almost impossible. So, if scar revision procedure were expected as a scar elimination one, the surgery may be considered disappointing. So, detailed consultation for those patients is the precious experience even from the basic plastic surgery training time. Also, both the surgeon and the patient must agree preoperatively that the goal of surgery is to improve not eliminate the scar to be satisfying management [3].

The cheek is relatively broad facial aesthetic unit which surrounds the central facial structures i.e. the nose and lips and fuses with forehead, eyelids, neck and ear with different reconstructive considerations. The cheek differs from men to women in unique characters which are of great aesthetic and reconstructive values. Homogeneous women cheeks are different from those covered with thicker hairbearing skin in men. The borders of the cheek subunit are composed of the nasofacial junction and nasolabial fold medially, the infraorbital rim superiorly, the temporal hairline and preauricular crease laterally, and the lower of the mandible inferiorly [4].

The facial skin exerts a sort of intrinsic tension which occurs in invisible lines named the relaxed skin tension lines (RSTL s) along which the incisions tend to heal in least tension and spreading with better aesthetic results. These lines occur in the face nearly paralel to rhytids lined. The cheek subunit borders and RSTLs provide the basis for proper scar positioning and orientation to maximize eventual scar camouflage [5]. Tissue expansion is an ideal strategy that generates a new skin with the same color, nature and characters of the surrounding healthy skin [6].

The motive of this study was that the results of some cheek scars revisions may appear to be 
disappointing owing to their sites or direction even with much efforts. By using tissue expanders, the scars repositioning and reorientation in relation to the cheek unit borders and RSTLs may provide the basis of superior results in scar revision and management.

So, we aim to demonstrate the effect of cheek skin expansion by tissue expanders on repositioning and reorientation of the cheek scar to maximize the scar aesthetic outcome.

\section{PATIENTS AND METHODS}

This prospective study was conducted on 13 patients with obvious linear (8 cases) or irregular (5 cases) cheek scars who were willing to move these scars to less conspicuous sites. In fact, the closest expandable skin to the scar area in one side is considered as the donor tissue. The scars included in the study must be in the cheek unit in a place permitting the insertion of a rectangular expander under the nearby haelthy skin. This potential donor skin must have the enough size and expandability to replace the required area in repositioning the scar. All patients included in the study have been undergone all the stages of the reconstruction from May 2013 to May 2017 with 6 months follow-up period at least in the unit of plastic and reconstructive surgery, Zagazig university hospitals. Adequate patients' consultation and written informed consents from all included patients were fullfiled.

We have designed a computerized custom-made chart (Fig. 1) including facial photographs of our patients to simulate face with the scar and tissue expander insertion site in a flat two-dimensional setting. We provisionally simulate the face pre, during and post expansion. For accurate analysis and planning, we have assigned the coordinates of three points in each scar (two ends and one midpoint).

Based on a previous study [7], rectangular tissue expanders are believed to have more expandability compared to crescent and round ones. Therefore, we used rectangular tissue expanders in all cases with (40 to $200 \mathrm{ml}$.) capacity. They were selected suitable to be inserted under the donor tissue and generate the proposed expansion.

At the day of expander insertion, preoperative marking of the original incision site, anatomical sites of vital structures and proposed subcutaneous pocket was done according to the previously planned chart paper.

\section{Operative technique:}

All surgeries were performed under general anesthesia. By scrub betadine the face was prepared followed by injection of adrenaline solution (from $1 / 100000$ to $1 / 200000$ ) in both the incision site and the pocket area. Limited skin incision $(3-6 \mathrm{~cm})$ is done at the edge of the scar nearby the pocket side and the subcutaneous pocket using fine skin hooks is done $10-20 \%$ larger than the tissue expander. It should be created below the donor area superficially to superficial musculaponeuotic system (SMAS). The use of sharp scissors with careful dissection and being under direct vision are the keystones for the technical safety. SMAS layer acts as a barrier to the plane containing facial nerve with its branches, parotid duct and other facial vital structures. Using bipolar diathermy, fine hemostasis was secured and the pocket area was washed with normal saline and antibiotic solution (160mg gentamicin and $500 \mathrm{ml}$ normal saline). After the expander was examined for safety and leakage, it was inserted in the pocket. During the insertion procedure, knuckling and bending of the prosthesis were carefully avoided. Also, the injection port was placed subcutaneously far from the pocket and the tissue expander under the healthy skin. It must be easily distinguishable and accessible for the following injections with avoidance of kinking and tension to insure successful expansion. Also, intraoperative examination for port performance should take place. Careful hemostasis was secured and vacuum drains were not used in any case but they were available to be placed in the pocket site if necessary. Then, using vicryl sutures, subcutaneous and deep dermal layers were closed. Subcuticular prolyne suture were taken afterwards to close the skin. Normal saline, $10 \%$ to $20 \%$ percent of the of the tissue expander volume, was injected as required. This induce an average pocket wall intrinsic compression with not much tension to the repaired wound. After the surgery, with the use of sterile gauze the wounds were dressed. According to the wound state, two to three weeks postoperatively, the expander inflation began and continued at 5-7 days intervals. The wound must be free of any sign of infection, inflammation or discontinuation to start the expander inflation.

It has been continued on average 2 to 3 months long according to the needs and the site of the surface area to reconstruct. We relayed on blanching of the skin and the patient discomfort to determine the safe filling endpoint indicator. To measure the tissue expansion, we were subtracting the width of the expander base from the width of the expanded skin on the expander. The expander inflation must be at least $10-20 \%$ more than the proposed size of the area planned to be excised to compensate the elastic recoil of the facial skin. Afterwards, we 
removed the expander in the second stage surgery. At this stage we excised the original scar and the planned nearby healthy skin to modify the wound place or direction. According to the availability, the expanded skin was used to shift the scar to more favorable site i.e. borders of the face, borders of the cheek subunit, to be reoriented in relaxed skin tension lines (RSTLs) or outside the malar region or in the hair bearing area. The incision site was repaired in two layers. (Fig. 2).

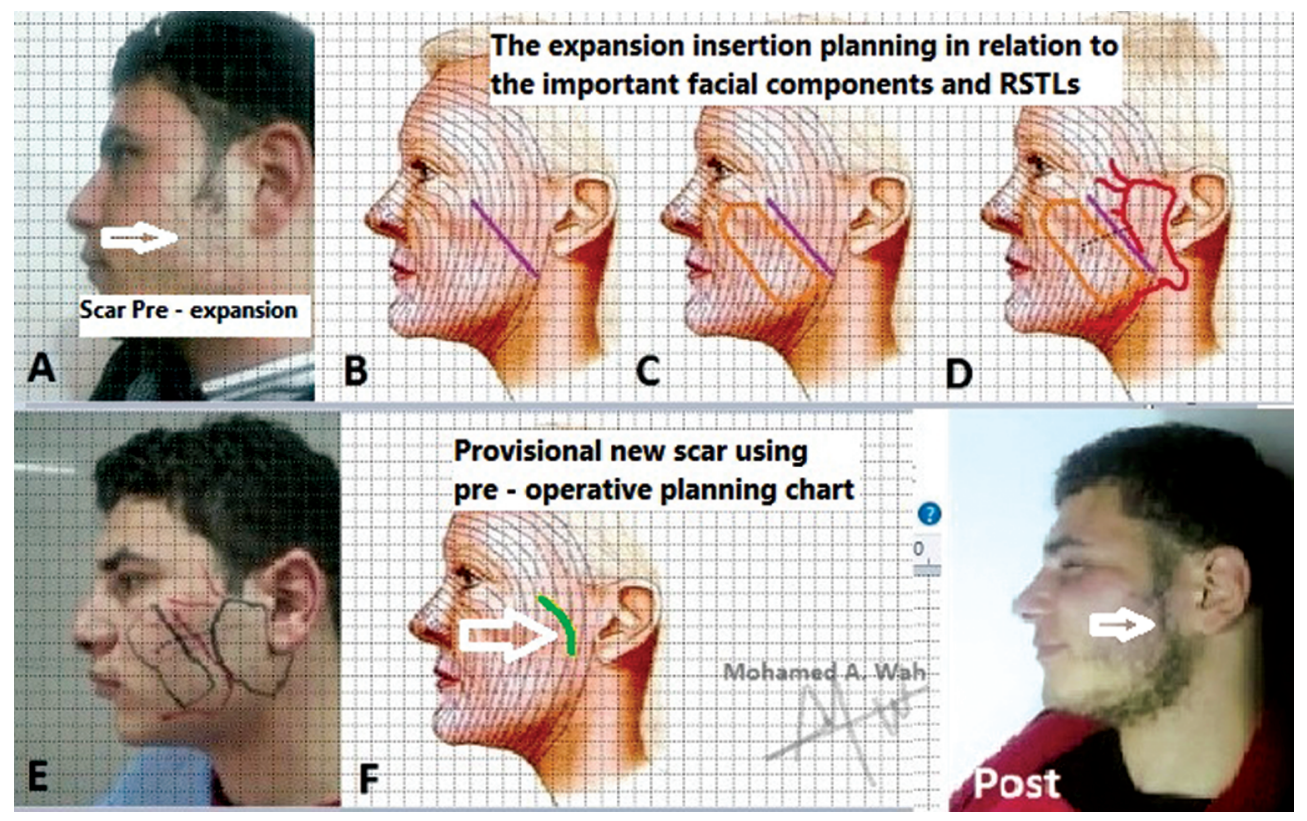

Fig. (1): Pre operative custom-made planning chart.

First Stage:

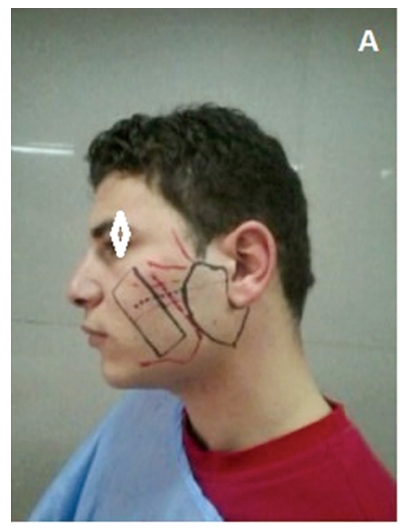

Second Stage:

(D): Expansion.

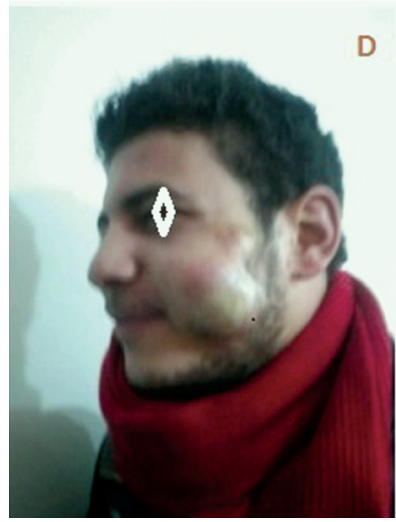

(B): Dissection.

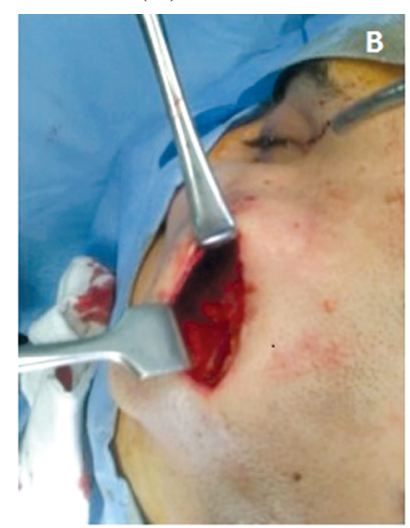

(E): Excision.

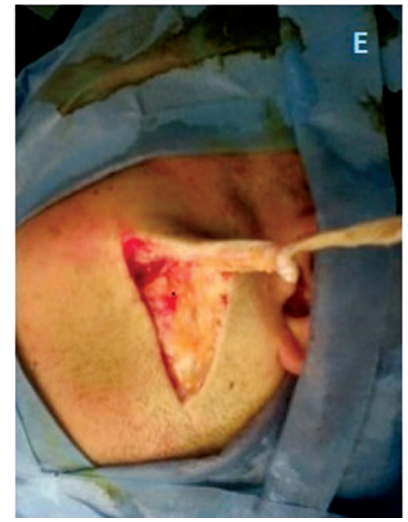

Fig. (2): Operative technique.
(C): Incrtion.

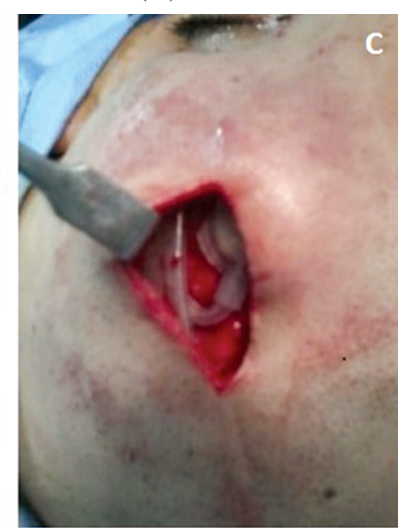

(F): Closure.

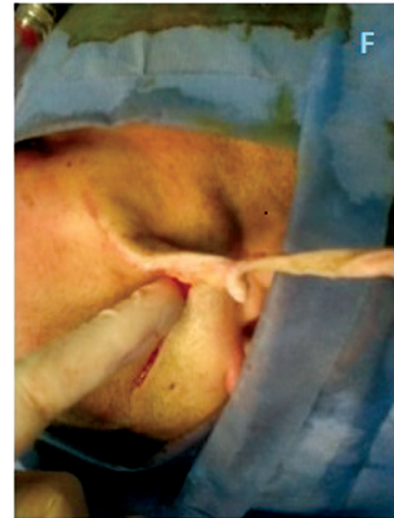


All the patients were analyzed and compared in terms of demographic variables, such as age and gender, etiology of scar, shape and the dimensions of the scar, the mean final volume of the prosthesis, the mean time interval between the two procedures.

Complications were assessed as major complications (infection, leakage, exposure, wound dehiscence, extrusion, and tissue necrosis) and minor complications (hematoma, seroma).

Regarding technical success, if no complications occurred, it was considered a "straight forward' expansion. Completion of expansion after treatment of complications was termed "salvage" while if the expansion had to be abandoned it was considered "failure".

For interpretation of the quantitative variables, age, filling volume, time of treatment. Standard deviation, mean, minimum, and maximum values were used.

For interpretation of the qualitative variables, three plastic surgeons not included in the study have aesthetically evaluated the scar pre-expansion and post-expansion to determine the aesthetic outcome i.e. complete repositioning the scar outside the cheek unit (optimal), scar reorientation typically in RSTLs or repositioning the scar outside the malar region (suboptimal), incomplete scar resection (partial) and the expansion was terminated without any scar resection (failed). Also, they evaluated homogeneity between the generated and the surrounding skin, facial symmetry with normal performance of the tissue and patient satisfaction at least 6 months after the reconstruction surgery and absolute frequency and relative frequency were used. The analyses were performed using the SPSS statistical software for windows (version 17.0 SPSS Inc., Chicago, IL, USA).

\section{RESULTS}

A total of 13 expanders were placed in 13 patients with cheek scars between May 2013 and December 2014. This series comprised 4 females and 9 males with a mean age 19.25 \pm 9.2 years (range: 11-31). Skin expansion was performed for patients of linear scars due to sharp injuries ( 7 males and one female) and irregular scars due to burns with boiling water ( 2 males and 3 females). Smooth surface rectangular expanders with a remote valve were used in the cheek aesthetic unit. These were classified according to the place of expander insertion (location of the majority of expander base surface area) into suborbital (3 cases), buccal (3 cases) and preauricular subunits ( 7 cases).

Rectangular expanders were used in the subcutaneous plane in all cases through an incision that was included in the scar to be removed. The volume of expanders used ranged between $40 \mathrm{ml}$ and $200 \mathrm{ml}$ (mean: $162 \mathrm{ml}$ ), while the fully expanded volumes ranged between 80 and $270 \mathrm{ml}$ (mean: 178ml). Time of treatment (time from expander placement to expander removal at the time of reconstruction ranged between 9 and 13 weeks with a mean of 10.2 weeks.

In 10 out of 13 cases (77\%) tissue expansion were achieved without complications. At the same time, one minor complication has occurred in one patient i.e. hematoma (resolved within one month) was termed "salvage". Major complications have occurred in 2 cases i.e. expanders were exposed. Since, in one case of these patients, this occurred during the final stages of expansion not affecting the outcome and termed "salvage". Only in one case, implant was withdrawn two weeks after insertion in the early period and it was termed "failure".

\section{Case 1:}

A 27 years old man presented with an aesthetically unpleasant scar interrupting the middle cheek aesthetic unit. It was because of sharp injury 3 years ago in a criminal accident (knife mark). Although the scar was not so wide, he was anxiously willing to move the scar more peripherally as he failed to find a job many times. In the first session, 150cc (rectangular) subcutaneous tissue expander was inserted in middle cheek regions. Expansion was continued for 10 weeks. The expanded flap allowed excision of the scar and the nearby skin shifting the scar $4 \mathrm{~cm}$ lateral, placing it more peripherally in the cheek within anterior hair line of the chin and outside malar region. (Fig. $3)$.

\section{Case 2:}

A 19 years old girl sustained a severe burn resulted in unpleasant scar in the lower lateral cheek region. She requested revision of her cheek scar. In first reconstruction session, 150cc (rectangular) tissue expander implant was inserted. Expansion was continued for 9 weeks. The saline volume instilled was $250 \mathrm{cc}$. In the second session, the expanded flap allowed excision of the $3 \mathrm{~cm}$ width of the cheek skin and reconstruction of the burnt area. (Fig. 4). 


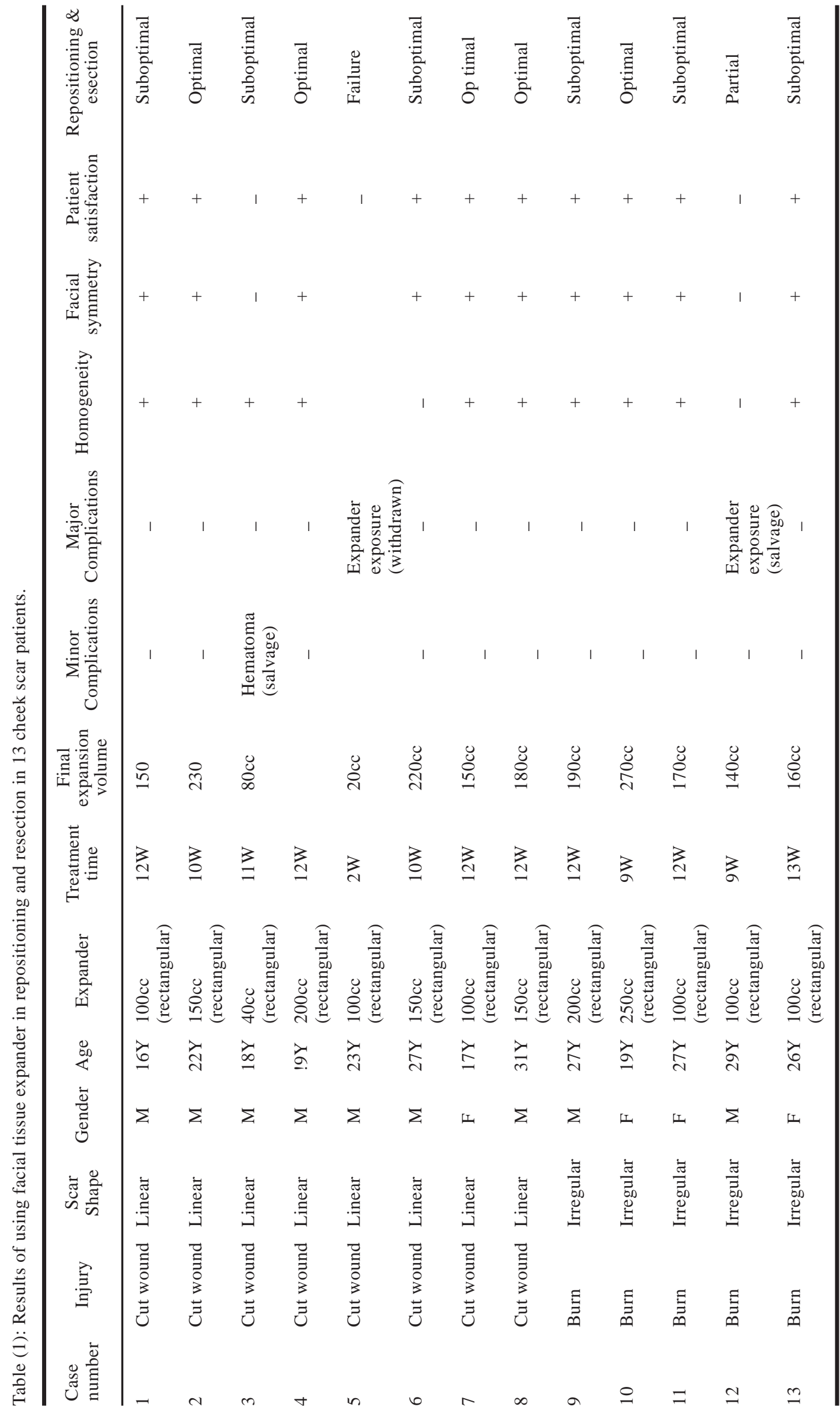




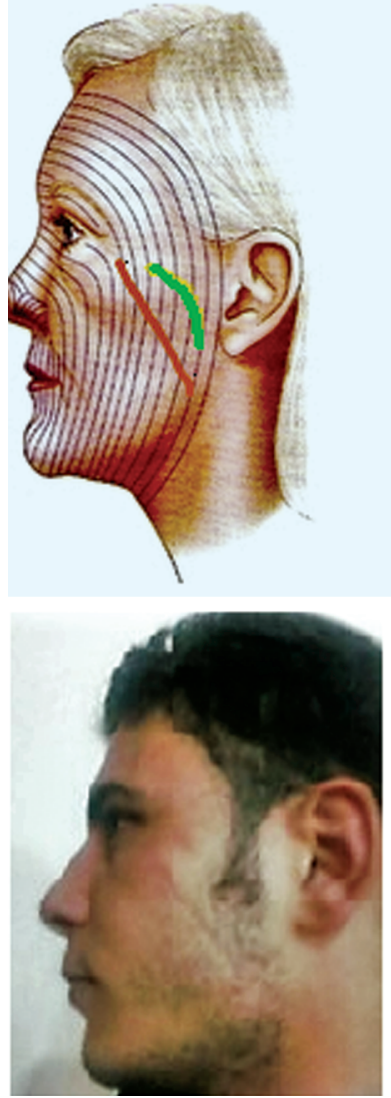

Pre-reposition
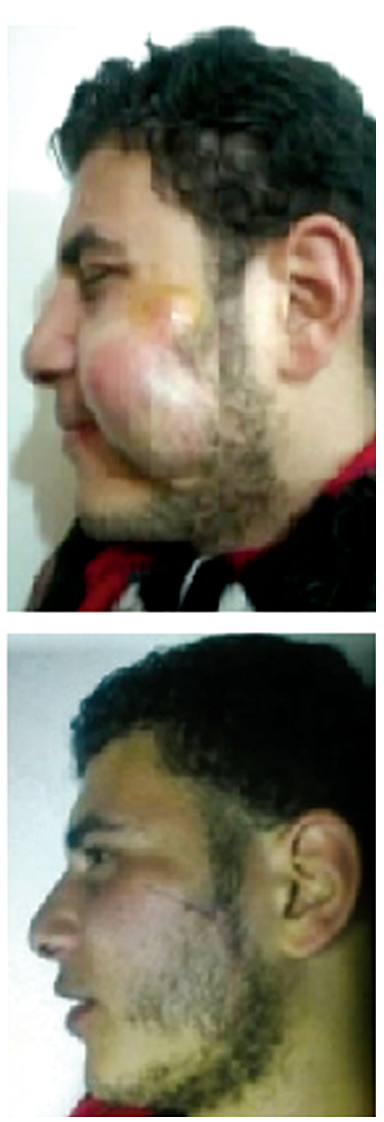

Post-reposition

Fig. (3 - Case 1): A 27 year old man presented with an aesthetically unpleasant scar interrupting the middle cheek aesthetic unit because of sharp injury 3 years ago in a criminal accident (knife mark).

\section{DISCUSSION}

Scar revision procedure(s) usually focuses on providing the scar the least conspicuous shape with satisfying long-term results. This may be still considered a challenge. Different options of facial scar revision have been routinely used to improve the unsightly scar to more ideal one. These techniques include excisional techniques i.e. simple fusiform excision with or without wound irregularization, serial excision and shave. Also, adjunctive techniques have been used like dermabrasion, laser resurfacing and intralesional steroid injection. Although all these options have provided a better aesthetic outcome, many patients and doctors still have a constant quest for seeking superior results. The skin has a great potential to expand by the effect of tension and so tissue expansion has been commonly used to generate a healthy skin to resect scars. The concept of reposition of an existing scar to a favorable site has been usually attempted in midface scar revision with undermining and excision techniques. However, this may result in some facial asymmetry and contour distortion.
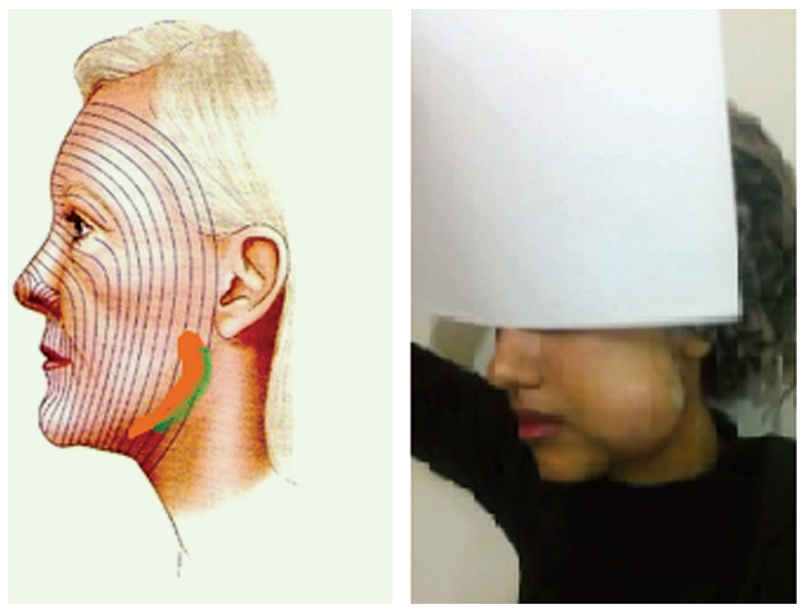

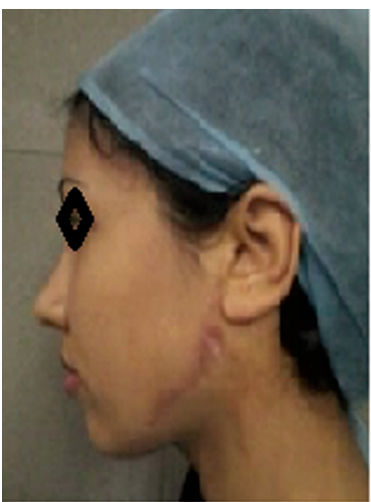

Pre-revision

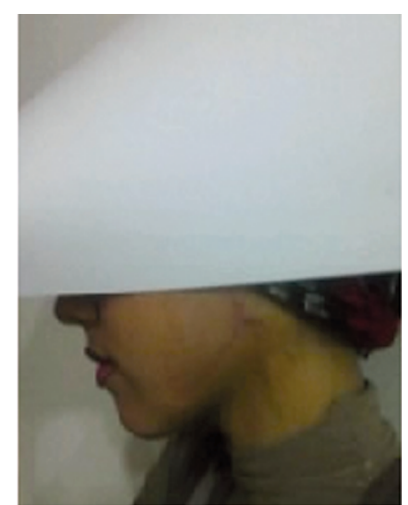

Post-revision
Fig. (4 - Case 2): A 19 year old girl sustained a severe burn resulted in unpleasant scar in the lower lateral cheek region. She requested reconstruction of her cheek.

In the present study, 13 cheek scars ( 8 secondary to cut wounds and 5 burn scars) were managed using tissue expanders. Tissue expansion in this study has been used to over expand the cheek skin and repositioning of the scars. So, it was primarily a repositioning plus excisional rather than a merely excisional technique. As a result, this approach was used in this literature to get rid of scars interrupting cheek aesthetic unit of the face or perpendicular to RSTL. Also, it avoided wound closure under tension with potential scar widening. Scar analysis in this literature has been achieved by printing the face photo on a computerized custom - made chart. This permitted better planning of the expander insertion site, the expansion needs and second stage design. Furthermore, it provided an efficient and clear tool and explain to the patients in more realistic consultation.

The mean age of the patients in our study was 19.25 \pm 9.2 (range: $11-31$ ) years. The mean age of the patients in the study by Motamed et al., was $25.5 \pm 8.3$ years. In the present study, 9 patients were males (69\%) and 4 patients were females 
$(31 \%)$. The types of the scars were linear scars $(62 \%)$ and irregular scars (38\%). The causes of these cheek scars were due to sharp injuries in the linear scars ( 7 males and one female) and boiling water burns in the irregular scars (2 males and 3 females). The majority of cases in the studies by Motamed et al., [13], Gao et al., [18] and Farahvash et al., [19] were females $(70.59 \%), 57.17 \%$ and $57 \%$ respectively. The high incidence of males in the present study compared to those studies is the higher inclusion of linear post sharp injuries scars which is seven folds commoner in males than females in our study. The cause of scar in the study by Ashab et al., was fire burn $(60.61 \%)$ followed by boiling water burn $(15.15 \%)$ in most of the cases [15]. In the series of Motamed et al. the most common cause was flame (47\%) and boiling water $(26.5 \%)$ [13]. This also may explain the majority of females' numbers than males in both of these studies who might be more subjected to burns in home accidents during food preparation with less safety precautions.

In the current study, all patients were operated using rectangular expanders. Rectangular expanders were found by Fattah to generate more tissue gain than of round or crescent expanders [7]. This has been supported by Hudson [8] and Motamed [9] with better flap design and coverage chances.

Facial tissue expansion procedures necessitate a special surgical experience. Tissue expanders insertion in head and neck have been usually used in reconstruction of extensively damaged tissues [9]. During searching literatures, managing the linear or small scars in the cheek using tissue expanders was found an uncommon practice. However, these scars may be a challenge in terms of leading a normal life, joining certain jobs or having a social and psychological balance. Complete elimination trials of these scars by local excisional and adjunctive techniques may be disappointing in some instances.

In the present study, optimal and suboptimal (beneficial) scar revisions were achieved in $84.6 \%$ of cases. The concept of complete moving the scar outside the cheek unit (optimal revision) has been applicable to 5 in 13 patients $(38.5 \%)$. However, suboptimal revision has been applicable in the form of scar reorientation typically in RSTLs succeeded in 5 of 13 patients $(38.5 \%)$ or repositioning the scar outside the malar region in one case $(7.7 \%)$. Partial revision in the form of incomplete scar resection occurred in 1 case $(7.7 \%)$. Only in one case $(7.7 \%)$, the expansion was aborted due to early wound dehiscence (failure). As regards to repositioning or reorientation of the linear cheek scars by tissue expansion, no literature was found to use the concept before this study according to my data. Based on the results achieved by burn scars of the current study, four in five cases (80\%) were completely reconstructed and one case (20\%) were incompletely resected. This is similar to the results found in the series of Fattah in 2014 [7], $80 \%$ of total patients suffering from head and neck burn scars have been reconstructed completely. Also, this is comparable to the results achieved by Hudson [8]. It is higher than that achieved by Bozkurt et al who had completely reconstructed $71.6 \%$ of their patients [11] but lower than the results of the study of Saleh et al., [12] $87.5 \% 12$ and Hafezi et al., [16] which were $90 \%$ of cases. In the current study, the results were approximate to the results found in previous studies $[\mathbf{7 , 8 , 1 1 , 1 2 , 1 6 ]}$ in spite of relatively difficult expansion of the cheek in our populations than the other areas of the head and neck $[7,10,12,16]$ and other body areas $[\mathbf{8 , 1 1}]$. Also, larger number of cases in future researches should be considered.

Generally, the insertion of tissue expander should be nearby the scar wherever the expansion site i.e. face, neck, scalp or body to generate a well-matched tissue with most similarity to the skin color and nature [14]. Based on our study data, tissue expanders were inserted in subcutaneous plane just on the SMAS layer of the cheek in all cases and the initial volume of the tissue expanders was between 40 and $200 \mathrm{ml}$. In different studies, the neck was the most common site for expander placement in cheek scar revision $[\mathbf{1 3 , 1 5 , 7 ]}$ with the largest applicable initial volumes. The mean initial volume by Yamin et al., was 321.55 \pm 182.52 [15]. In another study, the initial volume for reconstruction of cervicofacial angle was selected between 250 and 500ml [13]. In our series, marked over expansion was avoided above $20 \%$ more than planned preoperatively otherwise excessive skin may need excision with additional scar.

In the current study, two patients developed complications of the prosthesis $(15.4 \%)$, including, minor expander exposure $(7.7 \%)$ and one wound dehiscence $(7.7 \%)$. The patient who sustained of minor exposure was managed by continuation of expansion for 4 weeks to gain maximum possible skin expansion. The other case of wound dehiscence which was in the beginning of the expansion period was managed by prosthesis removal, wound lavage and immediate closure with antibiotic according to culture and sensitivity test. Limited hematoma was reported in one case $(7.7 \%)$ which was resolve within one month follow-up. This result was com- 
parable to the series of Yamin et al., who reported complications of prosthesis in $(13.89 \%)$ of his population [15]. The prosthetic complications reported by him were including prosthesis site infection $(2.78 \%)$, prosthesis exposure $(5.56 \%)$, hematoma $(2.78 \%)$, leakage of the prosthesis $(2.78 \%)$, and necrosis of the injection port site $(2.78 \%)$. In the present study, the total complication rate $(23 \%)$ which was lower to that reported by Hafezi et al., (27\%) [16] and Saleh et al., (34.75\%) [12] however comparable with that reported by Tavares et al. (24\%) [17] and Hudson (25\%) [8].

Bozkurt et al., [11] reported in there study approximately $30 \%$ complication rate. In the present study, the failure rate (interruption of expansion process) was $7.7 \%$. This is lower than that of Hudson (20\%) and Saleh (13.25\%) but comparable to that of Bozkurt et al., 10\% [11] and that of Hafezi et al., (6\%). We think that careful planning, meticulous dissection on subcutaneous plane above the SMAS, hemostasis and closure of dermal layer are the keystones of minimization the complication and failure rates.

\section{Conclusion:}

Facial tissue expander usage in repositioning and revision of linear and irregular cheek scar is a reasonable approach in obtaining superior aesthetic results.

\section{REFERENCES}

1- Matthew B. Klein.: Thermal, chemical and electrical injuries. Grabb \& Smith's "Plastic Surgery", 6 ${ }^{\text {th }}$ edition.

2- Siemionov M., Sönmez E. and Papay A.: Principles of facial transplantation. Weinzweig J. Plastic. Surgery. Secrets. Plus, $2^{\text {nd }}$ edition.

3- Rajeev B.A.: Scar revision is a useless operation: Opposing the statement. Indian J. Plastic Surg. January-June, Vol. 37 Issue 1: 25-27, 2004.

4- Steven D. Pletcher and David W. Kim: Current concepts in cheek reconstruction. Facial. Plast. Surg. Clin. N. Am. 13: 267-281, 2005.

5- Borges A.F.: Relaxed skin tension lines (RSTL) versus other skin lines. Plastic. Reconstr. Surg., 73: 144-50, 1984.
6- Rivera R., LoGiudice J. and Gosain A.K.: Tissue expansion in pediatric patients. Clin. Plast. Surg., 32: 35-44, 2005.

7- Fattah J.H.: Postburn head and neck reconstruction using tissue expanders. Zanco J. Med. Sci., 18 (3): 786-92, 2014.

8- Hudson A. and Grob M.: Optimizing results with tissue expansion: 10 simple rules for successful tissue expander insertion. BURNS, 31: 1-4, 2005.

9- Motamed S., Niazi F., Atarian S. and Motamed A.: Postburn head and neck reconstruction using tissue expanders. Burns., 34: 878-84, 2008.

10- Motamed S.1. and Fadaee-Naeeni A.: Reconstruction of the cheek, moustache, and beard by transferring an expanded cervical flap. Arch. Iran. Med., Mar., 11 (2): 2213, 2008.

11- Bozkurt A., Gregor A., Dey D. and Vogeler F.: Retrospective analysis of tissue expansion in reconstructive burn surgery: Evaluation of complication rates. BURNS, 34: 1113-8, 2008

12- Saleh Y.: Scalp reconstruction using tissue expander. Egypt J. Plast. Reconstr. Surg., 28: 71-5, 2004.

13- Motamed S. and Attarian S.H.: Cervicofacial angle reconstruction while insetting flaps to treat chronic burns. Pejouhandeh Shahid Beheshti Univ. Med. Sci., 14: 1, 2009.

14- Motamed S., Niazi F., Atarian S. and Motamed A.: Postburn head and neck reconstruction using tissue expanders. Burns, 34: 878-84, 2008.

15- Ashab Yamin M.R., Mozafari N., Mozafari M. and Razi Z.: Reconstructive Surgery of Extensive Face and Neck Burn Scars Using Tissue Expanders. World. J. Plast. Surg., 4 (1): 40-49, 2015.

16- Hafezi F., Naghibzadeh B. and Pegahmer M.: Use of overinflated tissue expanders in the surgical repair of head and neck scars. JPRAS, 20:1-8, 2008.

17- Tavares M., Belerique M., Franco D., Porchat A. and Franco T.: Tissue expansion in burn sequelae repair. Burns, 33: 246-51, 2007.

18- Gao J.H., Ogawa R., Hyakusoku H., Lu F., Hu Z.Q., Jiang P., Yang L. and Feng C.: Reconstruction of the face and neck scar contractures using staged transfer of expanded "Super-thin flaps". Burns, 33: 760-3, 2007.

19- Farah Vash M.R.: Tissue expander, a modality in the treatment of burn induced deformities: A 6-year study of patients with burn deformities admitted to Plastic Surgery Department of Imam Hospital. Tehran. Univ. Med. J., 58: $20-5,2000$. 